\title{
On-screen Note Pad for Creative Activities
}

\author{
Norikazu Iwamura, Kazuo Misue, and Jiro Tanaka \\ Department of Computer Science, \\ Graduate School of Systems and Information Engineering, University of Tsukuba, \\ 1-1-1 Tennoudai, Tsukuba, 305-8573 Japan \\ \{iwamura, misue, jiro\} @iplab.cs.tsukuba.ac.jp
}

\begin{abstract}
We propose a system that enables users to write memos directly on a computer screen by hand. The system helps users save the screen images along with a user's handwritten strokes and in searching for them. We describe some of the important functions of the system and the effective retrieval keys for searching for saved images. We explain that the implementation of our prototype system consists of two subsystems: note-taking and browsing systems. An example of the usage of the system is also described.
\end{abstract}

\section{Introduction}

Currently, various types of researches are being conducted on computers. We are often working with intellectually creating ideas, and there are a lot of situations where we unconsciously hit upon some new ideas. For example, when researchers are browsing the web, they might hit upon an idea for their new research, or when a designer is looking at pictures on a PC, the idea for a new design might by chance be realized. However, when the idea presents itself, it might not be able to be put immediately into practice. In many cases, after some time is put into it, it will be reflected. Therefore, taking notes is generally done as a way of saving ideas.

When we are using a computer, using a pen and paper is currently the easiest way to take notes. This is the widely used means of saving ideas, because users can take notes quickly by hand. If there are materials that have been printed out on paper, it is possible to freely write on them, and the relation to the content of the paper can be written at the same time. However, if neither paper nor pen is available, it is impossible to take notes. It takes time to print out the paper and use it. Therefore, we often temporarily take notes on the computer.

It is possible to save notes as text data using word-processing software (note pad and word pad, etc.) to save information on the computer. However, because word-processing software can only save text data, the user's idea cannot be properly reflected using this method. Moreover, when the user starts the application to write notes, a new window opens. This new window hides the original information that brought about the user's idea because it uses a different application, making it possible for the user to forget their original idea.

Our purpose is to support "writing memos". We are developing a system where users can take notes on the computer screen, just like they would on paper. This system would help users save their ideas and helps in the reproduction of those ideas. 
We have also considered some of the functions that would be necessary to achieve this purpose, and have developed a prototype system with these functions.

\section{Overview of on-screen note pad}

We aim to make a note pad system that gives users the feeling that they are writing on paper, and supports users in using saved notes with the advantage of a computer system.

\subsection{Natural Interface like paper}

Paper is advantageous to users because it can be easily and simply used. We propose a function where a user can use our system like they use paper.

\section{Starting without disturbing thinking process}

If users must click an icon to start a system, users must be conscious of the application switch, and it is necessary to display the icon on the screen. The application switching might bring about the disturbance of a user's idea. We adopted a start method that uses either the mouse or a shortcut key. Therefore, users can start the system without disturbing their thought process because the displayed screen doesn't change.

\section{Writing notes on computer screen using handwritten input}

This system adopts a pen interface and the use of handwritten input. With handwritten input, users can freely write characters and construct figures just like on paper. Users often have a lot of vague broken ideas, and saving them by handwritten input is very useful for collecting them.

It is possible to write notes just like on paper. This system uses a displayed screen as the window background, and the user writes strokes on that window. The relationship between the information on a stroke position and the screen image can be used for writing notes.

\subsection{Advanced functions beyond paper}

A computer system has its own advantages that differ from paper, such as using saved notes as data. We will describe some functions that make use of these advantages in this section.

\section{Browsing notes}

A browsing system is necessary to look over saved notes. When users browse notes, they expect the note that was used the most to probably be the one saved last. When 
users start up their systems, the memo that was saved last should be the one that is displayed.

When a user wants to read saved notes, they go through a lot of trouble if they have to check their saved notes one by one. This system displays a lot of notes at the same time using a thumbnail format. When using paper, it might be possible for information on paper to be hidden by a large amount of strokes. It is possible for this system to display only the screen image without the strokes, because it saves the stroke and screen image data separately.

To maintain a user's idea, this system can reproduce strokes according to the time they were written. This system can reproduce the order and speed of strokes similar to the time that the user took the notes, so the user's initial impression can be effectively reproduced. Users can not only browse the saved memo but also edit it, and the saved memo can be expanded. Since the edited notes might have greatly changed the original ones, they are treated as another new note.

\section{Searching notes}

It is necessary to efficiently search the stored notes that users want to retrieve to effectively exploit the notes. Therefore, some retrieval functions should be prepared. The keys to the retrieval functions should be their effectiveness at searching for the notes and being user-friendly. We use two kinds of retrieval keys. We will describe these retrieval keys in more detail in the next section.

\subsection{Retrieval keys}

We took into consideration the time and stroke information for the retrieval keys. The stroke information has the advantage in that the stroke impressions remain because the users write by hand. Therefore, it is advantageous for the users to use the stroke information as one of the retrieval keys. However, it is difficult to obtain a highly accurate retrieval using the stroke information, because the strokes that the user writes as the retrieval keys are vaguely shaped. On the other hand, the time information has the advantage that retrieval is easy from a technical point of view because it is exact numerical information. However, the load needed to remember the accurate time might be too large for users. We thought that the retrieval could work effectively by using both of these advantages together.

\section{Time information}

One of the simplest types of time information that can be used as a retrieval key is the time that a memo was stored. We can use a retrieval method that uses the length of note-taking time by counting the time from when the user began taking notes to when they finished taking notes. A retrieval method based on a user's memory might also be possible considering the length of time needed to take the notes. For example, users can use their memory of consideration how worried the user was when they were writing notes, or whether they took the notes concisely or not. However, a users' memory might not be accurate enough when searching for notes using time information. Therefore, attempting exact matching using time information is not 
useful to users, we should allow some of the ambiguities found in matching using time information keys.

\section{Stroke information}

The properties of written strokes are also effective as retrieval keys. We can obtain the amount of information that was written by examining the number and length of the written strokes. We can judge how intensively the user wrote during a unit of time by using the time and stroke information.

The shape of a stroke can also be used as a retrieval key. When users take notes, it can be expected that the annotation is often applied because users can use the screen image as a window background. To use such information, strokes are analyzed and used as retrieval keys. Figure 1 shows some examples. The shapes of strokes, like the examples shown in Figure 1, are counted by an analyzing system, and users can retrieve notes according to the numbers. However, there is vagueness because strokes are written by handwritten input. The shape of stroke like strokes shown in Figure 1 might not be written. When users retrieve information, some analytical results that have a high-level of similarity are also shown. If the similarity level is higher, the retrieval hit rate is higher.

\section{情報を閱覧}

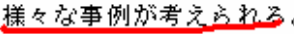

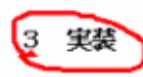

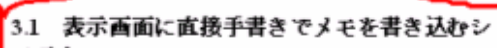$$
\text { ステム }
$$
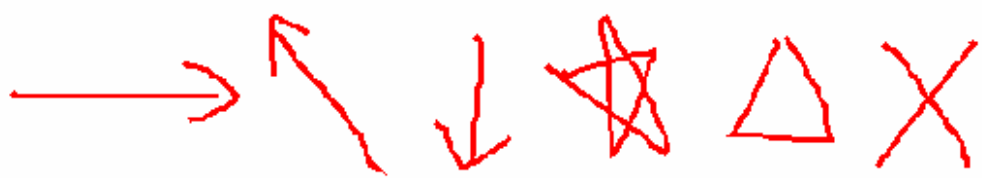

Figure 1: Examples of shapes used as retrieval keys

(Underlines, inclusions, arrows, and marks, etc.)

\section{Prototype implementation}

We have implemented a prototype system called "Leafletnote" to achieve the functions that are proposed in Section 2. The system consists of two subsystems: a note-taking subsystem and a browsing subsystem. 


\subsection{Note-taking system}

With the note-taking system, users can write strokes on the displayed screen by hand, and save the strokes with the screen image.

Figure 2 shows a screen image of the note-taking system. When a user starts the system, they can freely write strokes on the screen by hand. When they are finished taking notes, they just tap the screen. Then the system saves the memo in the save folder, and the system shuts down.

Figure 3 shows the explanation of making a starting window. When this system is started, the system captures the screen image on the display at that time, and shows the image data on the window. So, the user can operate the window with the background maintained.

Users can write strokes by hand on this window. Therefore, we have achieved the ability to write directly on the display.

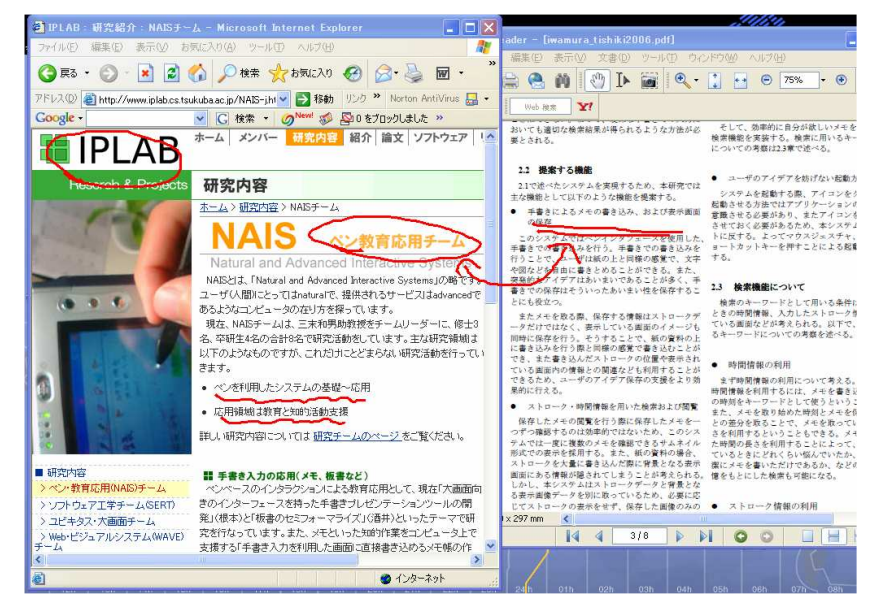

Figure 2: Example of Note-taking system image

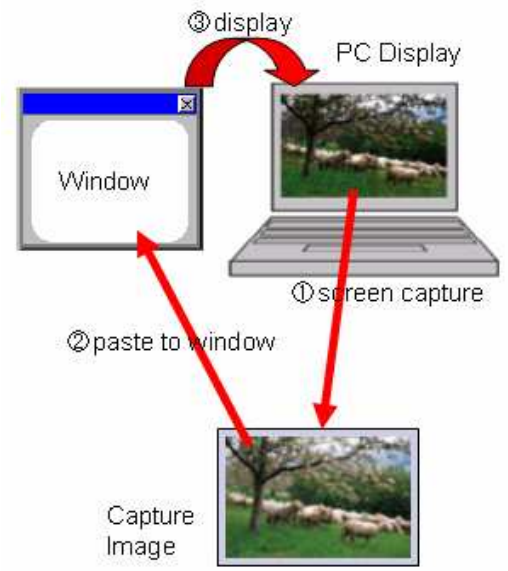

Figure 3: Explanation of making starting window 


\subsection{Browsing system}

The browsing system helps the users retrieve notes stored with the note-taking system and helps to remind them of their ideas.

Figure 4 shows an image of a browsing system. When a user starts this system, the note that was most recently saved is displayed in the largest frame. If the user clicks one of the thumbnails on the left side of the window, the note that corresponds to the thumbnail is displayed in the large section on the right side of the window.

The stroke and image data are saved separately. So, it is possible to display only the screen image without the strokes. This function enables users to see the original screen images at any time. The time and coordinate data are also saved with the stroke data. This data can be used to replay the writing of the strokes. This function helps users to remember the situation in which they wrote the strokes. They can use these functions by selecting items from the menu.

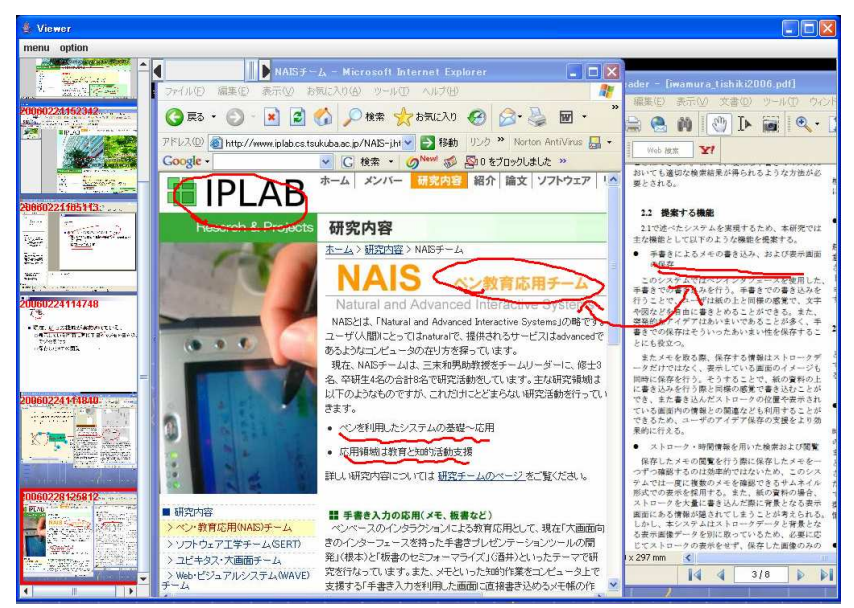

Figure 4: Example of browsing system image

\subsection{Implementation environment}

We used the JAVA language for implementing the system. We used the making support tool kit SATIN [1] for the pen-based application. The processing and analysis of the stroke data, and the drawing canvas were used from the SATIN library.

\section{Example of system application}

In this section we will describe a concrete scene of the use of this system. We assume a situation where users were performing research on their areas of interest on their computers. The following examples are imaginable: 
1. When the users find research papers related to their area of interest on the Internet, they read the papers on their computer screens.

2. When they find important information in the papers and hit on some ideas, they start the note-taking system by pushing a shortcut key. This operation does not disturb their activities. They write notes, make marks, or underline items on the interesting area of the display screen. When they finish writing notes, they tap the screen and the system saves the notes with the screen image.

3. While they are reading the research papers, if they want to know the details concerning the research topics, they open the related web pages and study the detailed information. By using this system, they can write notes while displaying both the research paper and the web page.

4. They look at the saved note pad later. They can look over the content of the web pages and research papers that they saw before, because the screen image and written strokes are saved in the notes, making it is easier to remember their idea. They can review their strokes by using the reproduction function, and reconfirm the image when writing a note. If they hit on more ideas, they can add their ideas to the note, and the content of the note is expanded.

5. When they want to look over a note that was saved in the past, they use a retrieval function. At that time, they can find the notes by using some vague information (stroke, time, and so on), even if their memory is unclear.

\section{$5 \quad$ Related Works}

Researches that aim to support the thinking process or for taking notes by hand have been conducted in the past.

ScreenCrayons [2] is common to our research as it targets the saving of written text by hand. This system provides a function that is able to write the annotation in various applications, and to save the part in which the annotation was written as an image. However, the saved object is only a part of the image where the annotation was written. We aim to support the user's intellectual creation by using information on the background. Electronic Cocktail Napkin [3] is a system that can freely take handwritten notes. However, it differs from our system in that the displayed image cannot be used as a background image. InkDesktop [4] and PenPlus pro [5] are systems that can write in the displayed screen using handwritten pen input, but they don't support how to use the saved notes. SmartCalendar/SmartWrite [6] is a system that aims to take notes using handwritten input. It has capture screen and retrieval functions using time information, but it does not have a retrieval function using the stroke information.

Kamicopi [7] is a system that aims to save data and reuse it. It takes data from web pages and e-mail and so on, and freely reuses it. However, users cannot use handwritten input in this system. Microsoft OneNote [8] has a note pad function that has been enhanced. This software can be used with data from various media and applications, and memos can be written by hand. However, there is the problem of 
hidden background images when the new window opens. Another problem is the operation might be too complex because the system provides various functions.

\section{Conclusion and future work}

We have proposed and implemented a note pad system that was able to write notes directly on a computer screen by hand. Users can save the screen image and handwritten strokes with this note-taking system. They can use a saved note and reproduce saved strokes using a browsing system.

There are some future works that we are planning. We plan to implement the retrieval function and keyboard input to enhance our prototype system. We will consider some natural interfaces, for example, whether the "tap" method to save notes is appropriate or not.

Users who will actually use the system will be used to evaluate the effectiveness of the system, and we will investigate the frequency of use and the users' satisfaction ratings (about input, inspection, and retrieval phase).

\section{References}

1. J. Hong and J. A. Landay, 2000. SATIN: A Toolkit for Informal Ink-based Applications. In Proceedings of User Interface Software and Technology: UIST 2000, pp.63-72.

2. Dan R. Olsen Jr., Trent Taufer, and Jerry Alan Fails. ScreenCrayons: Annotating Anything, In Proceedings of User Interface Software and Technology: UIST 2004 pp.165-174.

3. M. D. Gross, and E. Do. Demonstrating the Electronic Cocktail Napkin: a paper-like interface for early design, Conference Companion, ACM Conference on Human Factors in Computing: CHI 96, pp.5-6.

4. Microsoft: Microsoft Windows XP Tablet PC Edition http://www.microsoft.com/japan/windowsxp/tabletpc/downloads/enhancementpack/default. mspx

5. PlusSoft: PenPlus pro http://www.plussoft.co.jp/penplus/pro/

6. Kaoru Misaki and Naoki Kato. SmartWrite: Peperlike pen-based memo tool pursued brevity, In Proceedings of Workshop on Interactive Systems and software 2005 (WISS 2005), pp. 37 - 42. Japan Society for Software Science and Technology (in Japanese)

7. kamilabo.jp: Kamicopi http://www.kamilabo.jp/copi/index.html

8. Microsoft: OneNote http://www.microsoft.com/japan/office/onenote/prodinfo/default.mspx 